\title{
Representing by Orthogonal Polynomials for Sums of Finite Products of Fubini Polynomials
}

\author{
Dae San Kim ${ }^{1}{ }^{1}$, Dmitry V. Dolgy ${ }^{2}$, Dojin Kim ${ }^{3, *}$ and Taekyun Kim ${ }^{4}$ \\ 1 Department of Mathematics, Sogang University, Seoul 04107, Korea; dskim@sogang.ac.kr \\ 2 Kwangwoon Institute for Advanced Studies, Kwangwoon University, Seoul 01897, Korea; d_dol@mail.ru \\ 3 Department of Mathematics, Pusan National University, Busan 46241, Korea \\ 4 Department of Mathematics, Kwangwoon University, Seoul 01897, Korea; tkkim@kw.ac.kr \\ * Correspondence: kimdojin@pusan.ac.kr
}

Received: 12 March 2019; Accepted: 27 March 2019; Published: 29 March 2019

\begin{abstract}
In the classical connection problem, it is dealt with determining the coefficients in the expansion of the product of two polynomials with regard to any given sequence of polynomials. As a generalization of this problem, we will consider sums of finite products of Fubini polynomials and represent these in terms of orthogonal polynomials. Here, the involved orthogonal polynomials are Chebyshev polynomials of the first, second, third and fourth kinds, and Hermite, extended Laguerre, Legendre, Gegenbauer, and Jabcobi polynomials. These representations are obtained by explicit computations.
\end{abstract}

Keywords: fubini polynomials; orthogonal polynomials; Chebyshev polynomials; Hermite polynomials; extended laguerre polynomials; Legendre polynomials; Gegenbauer polynomials; Jabcobi polynomials

\section{Introduction and Preliminaries}

In this section, after fixing some notations, we will state the necessary basic facts on orthogonal polynomials as minimum as possible. For more details on the fascinating realm of orthogonal polynomials, the readers may refer to some standard books on those subjects, for example $[1,2]$.

The falling factorial polynomials $(x)_{n}$ and the rising factorial polynomials $\langle x\rangle_{n}$ for any nonnegative integer $n$, are given by

$$
(x)_{0}=1, \quad(x)_{n}=x(x-1) \cdots(x-n+1), \quad(n \geq 1)
$$

and

$$
\langle x\rangle_{0}=1, \quad\langle x\rangle_{n}=x(x+1) \cdots(x+n-1), \quad(n \geq 1),
$$

respectively. The two factorial polynomials in (1) and (2) satisfy the following relations:

$$
\begin{aligned}
& (-1)^{n}(x)_{n}=\langle-x\rangle_{n}, \quad(-1)^{n}\langle x\rangle_{n}=(-x)_{n}, \quad(n \geq 0), \\
& \frac{\Gamma(x+n)}{\Gamma(x)}=\langle x\rangle_{n}, \quad \frac{\Gamma(x+1)}{\Gamma(x+1-n)}=(x)_{n}, \quad(n \geq 0),
\end{aligned}
$$

where $\Gamma(x)$ is the gamma function. Also, the hypergeometric function ${ }_{p} F_{q}$ is defined by

$$
{ }_{p} F_{q}\left(a_{1}, \cdots, a_{p} ; b_{1}, \cdots, b_{q} ; x\right)=\sum_{n=0}^{\infty} \frac{\left\langle a_{1}\right\rangle_{n} \cdots\left\langle a_{p}\right\rangle_{n}}{\left\langle b_{1}\right\rangle_{n} \cdots\left\langle b_{q}\right\rangle_{n}} \frac{x^{n}}{n !} .
$$


Next, we will provide some basic facts about Chebyshev polynomials of the first kind $T_{n}(x)$, second kind $U_{n}(x)$, third kind $V_{n}(x)$, and fourth kind $W_{n}(x)$. Further, we refer to those facts about Hermite polynomials $H_{n}(x)$, extended Laguerre polynomials $L_{n}^{\alpha}(x)$, Legendre polynomials $P_{n}(x)$, Gegenbauer polynomials $C_{n}^{(\lambda)}(x)$, and Jacobi polynomials $P_{n}^{(\alpha, \beta)}(x)$. All of these facts can be found also in [3-9].

The above-mentioned polynomials are explicitly given as in the following:

$$
\begin{aligned}
& T_{n}(x)={ }_{2} F_{1}\left(-n, n ; \frac{1}{2} ; \frac{1-x}{2}\right) \\
& =\frac{n}{2} \sum_{l=0}^{\left[\frac{n}{2}\right]}(-1)^{l} \frac{1}{n-l}\left(\begin{array}{c}
n-l \\
l
\end{array}\right)(2 x)^{n-2 l}, \quad(n \geq 1), \\
& U_{n}(x)=(n+1){ }_{2} F_{1}\left(-n, n+2 ; \frac{3}{2} ; \frac{1-x}{2}\right) \\
& =\sum_{l=0}^{\left[\frac{n}{2}\right]}(-1)^{l}\left(\begin{array}{c}
n-l \\
l
\end{array}\right)(2 x)^{n-2 l}, \quad(n \geq 0), \\
& V_{n}(x)={ }_{2} F_{1}\left(-n, n+1 ; \frac{1}{2} ; \frac{1-x}{2}\right) \\
& =\sum_{l=0}^{n}\left(\begin{array}{c}
2 n-l \\
l
\end{array}\right) 2^{n-l}(x-1)^{n-l}, \quad(n \geq 0), \\
& W_{n}(x)=(2 n+1)_{2} F_{1}\left(-n, n+1 ; \frac{3}{2} ; \frac{1-x}{2}\right) \\
& =(2 n+1) \sum_{l=0}^{n} \frac{2^{n-l}}{2 n-2 l+1}\left(\begin{array}{c}
2 n-l \\
l
\end{array}\right)(x-1)^{n-l}, \quad(n \geq 0), \\
& H_{n}(x)=n ! \sum_{l=0}^{\left[\frac{n}{2}\right]} \frac{(-1)^{l}}{l !(n-2 l) !}(2 x)^{n-2 l}, \quad(n \geq 0), \\
& L_{n}^{(\alpha)}(x)=\frac{\langle\alpha+1\rangle_{n}}{n !}{ }_{1} F_{1}(-n ; \alpha+1 ; x) \\
& =\sum_{l=0}^{n} \frac{(-1)^{l}\left(\begin{array}{c}
n+\alpha \\
n-l
\end{array}\right)}{l !} x^{l}, \quad(\alpha>-1, n \geq 0), \\
& P_{n}(x)={ }_{2} F_{1}\left(-n, n+1 ; 1 ; \frac{1-x}{2}\right) \\
& =\frac{1}{2^{n}} \sum_{l=0}^{\left[\frac{n}{2}\right]}(-1)^{l}\left(\begin{array}{c}
n \\
l
\end{array}\right)\left(\begin{array}{c}
2 n-2 l \\
n
\end{array}\right) x^{n-2 l}, \quad(n \geq 0), \\
& C_{n}^{(\lambda)}(x)=\left(\begin{array}{c}
n+2 \lambda-1 \\
n
\end{array}\right){ }_{2} F_{1}\left(-n, n+2 \lambda ; \lambda+\frac{1}{2} ; \frac{1-x}{2}\right) \\
& =\sum_{k=0}^{\left[\frac{n}{2}\right]}(-1)^{k} \frac{\Gamma(n-k+\lambda)}{\Gamma(\lambda) k !(n-2 k) !}(2 x)^{n-2 k}, \quad\left(\lambda>-\frac{1}{2}, \lambda \neq 0, n \geq 0\right), \\
& P_{n}^{(\alpha, \beta)}(x)=\frac{\langle\alpha+1\rangle_{n}}{n !}{ }_{2} F_{1}\left(-n, 1+\alpha+\beta+n ; \alpha+1 ; \frac{1-x}{2}\right) \\
& =\sum_{k=0}^{n}\left(\begin{array}{c}
n+\alpha \\
n-k
\end{array}\right)\left(\begin{array}{c}
n+\beta \\
k
\end{array}\right)\left(\frac{x-1}{2}\right)^{k}\left(\frac{x+1}{2}\right)^{n-k},(\alpha, \beta>-1, n \geq 0) .
\end{aligned}
$$

We recall here Rodrigues-type formulas for Hermite and extended Laguerre polynomials, and Rodrigues' formulas for Chebyshev polynomials of all kinds and Legendre, Gegenbauer and Jacobi polynomials. 


$$
\begin{aligned}
T_{n}(x) & =\frac{(-1)^{n} 2^{n} n !}{(2 n) !}\left(1-x^{2}\right)^{\frac{1}{2}} \frac{d^{n}}{d x^{n}}\left(1-x^{2}\right)^{n-\frac{1}{2}}, \\
U_{n}(x) & =\frac{(-1)^{n} 2^{n}(n+1) !}{(2 n+1) !}\left(1-x^{2}\right)^{-\frac{1}{2}} \frac{d^{n}}{d x^{n}}\left(1-x^{2}\right)^{n+\frac{1}{2}}, \\
(1-x)^{-\frac{1}{2}}(1+x)^{\frac{1}{2}} V_{n}(x) & =\frac{(-1)^{n} 2^{n} n !}{(2 n) !} \frac{d^{n}}{d x^{n}}(1-x)^{n-\frac{1}{2}}(1+x)^{n+\frac{1}{2}}, \\
(1-x)^{\frac{1}{2}}(1+x)^{-\frac{1}{2}} W_{n}(x) & =\frac{(-1)^{n} 2^{n} n !}{(2 n) !} \frac{d^{n}}{d x^{n}}(1-x)^{n+\frac{1}{2}}(1+x)^{n-\frac{1}{2}}, \\
H_{n}(x) & =(-1)^{n} e^{x^{2}} \frac{d^{n}}{d x^{n}} e^{-x^{2}}, \\
L_{n}^{\alpha}(x) & =\frac{1}{n !} x^{-\alpha} e^{x} \frac{d^{n}}{d x^{n}}\left(e^{-x} x^{n+\alpha}\right), \\
P_{n}(x) & =\frac{1}{2^{n} n !} \frac{d^{n}}{d x^{n}}\left(x^{2}-1\right)^{n}, \\
\left(1-x^{2}\right)^{\lambda-\frac{1}{2}} C_{n}^{(\lambda)}(x) & =\frac{(-2)^{n}}{n !} \frac{\langle\lambda\rangle_{n}}{\langle n+2 \lambda\rangle_{n}} \frac{d^{n}}{d x^{n}}\left(1-x^{2}\right)^{n+\lambda-\frac{1}{2}}, \\
(1-x)^{\alpha}(1+x)^{\beta} P_{n}^{(\alpha, \beta)}(x) & =\frac{(-1)^{n}}{2^{n} n !} \frac{d^{n}}{d x^{n}}(1-x)^{n+\alpha}(1+x)^{n+\beta} .
\end{aligned}
$$

As is well known, the orthogonal polynomials in (6)-(14) satisfy the following orthogonalities with respect to various weight functions:

$$
\int_{-1}^{1}\left(1-x^{2}\right)^{-\frac{1}{2}} T_{n}(x) T_{m}(x) d x=\frac{\pi}{\epsilon_{n}} \delta_{n, m},
$$

where

$$
\begin{aligned}
& \epsilon_{n}=\left\{\begin{array}{ll}
1, & \text { if } n=0, \\
2, & \text { if } n \geq 1,
\end{array} \quad \delta_{n, m}= \begin{cases}0, & \text { if } n \neq m, \\
1, & \text { if } n=m .\end{cases} \right. \\
& \int_{-1}^{1}\left(1-x^{2}\right)^{\frac{1}{2}} U_{n}(x) U_{m}(x) d x=\frac{\pi}{2} \delta_{n, m}, \\
& \int_{-1}^{1}\left(\frac{1+x}{1-x}\right)^{\frac{1}{2}} V_{n}(x) V_{m}(x) d x=\pi \delta_{n, m}, \\
& \int_{-1}^{1}\left(\frac{1-x}{1+x}\right)^{\frac{1}{2}} W_{n}(x) W_{m}(x) d x=\pi \delta_{n, m}, \\
& \int_{-\infty}^{\infty} e^{-x^{2}} H_{n}(x) H_{m}(x) d x=2^{n} n ! \sqrt{\pi} \delta_{n, m}, \\
& \int_{0}^{\infty} x^{\alpha} e^{-x} L_{n}^{\alpha}(x) L_{m}^{\alpha}(x) d x=\frac{1}{n !} \Gamma(\alpha+n+1) \delta_{n, m}, \\
& \int_{-1}^{1} P_{n}(x) P_{m}(x) d x=\frac{2}{2 n+1} \delta_{n, m}, \\
& \int_{-1}^{1}\left(1-x^{2}\right)^{\lambda-\frac{1}{2}} C_{n}^{(\lambda)}(x) C_{m}^{(\lambda)}(x) d x=\frac{\pi 2^{1-2 \lambda} \Gamma(n+2 \lambda)}{n !(n+\lambda) \Gamma(\lambda)^{2}} \delta_{n, m}, \\
& \int_{-1}^{1}(1-x)^{\alpha}(1+x)^{\beta} P_{n}^{(\alpha, \beta)}(x) P_{m}^{(\alpha, \beta)}(x) d x \\
& =\frac{2^{\alpha+\beta+1} \Gamma(n+\alpha+1) \Gamma(n+\beta+1)}{(2 n+\alpha+\beta+1) \Gamma(n+\alpha+\beta+1) \Gamma(n+1)} \delta_{n, m} .
\end{aligned}
$$


The Fubini polynomials $F_{n}(y)$ are given (see, [10-13]), in terms of generating function, by

$$
G(t, y)=\frac{1}{1-y\left(e^{t}-1\right)}=\sum_{n=0}^{\infty} F_{n}(y) \frac{t^{n}}{n !},
$$

Then, from (34), it is easy to see that

$$
F_{n}(y)=\sum_{l=0}^{n} S_{2}(n, l) l ! y^{l}
$$

where $S_{2}(n, l)$ are the Stirling numbers of the second kind (see, [10-13]).

Note here that $E_{n}=F_{n}\left(-\frac{1}{2}\right)$ are the Euler numbers given by

$$
\frac{2}{e^{t}+1}=\sum_{n=0}^{\infty} E_{n} \frac{t^{n}}{n !}
$$

In this paper, we are going to investigate the following sums of products of Frobenious polynomials and express them as linear combinations of nine orthogonal polynomials in (6)-(14) (see Theorem 1):

$$
\alpha_{m, r}(y)=\sum_{i_{1}+\cdots+i_{r+1}=m}\left(\begin{array}{c}
m \\
i_{1}, \cdots, i_{r+1}
\end{array}\right) F_{i_{1}}(y) \cdots F_{i_{r+1}}(y), \quad(m, n \geq 0),
$$

where the summation runs over all nonnegative integers $i_{1}, \cdots, i_{r+1}$ with $i_{1}+\cdots i_{r+1}=m$, and we note that $\alpha_{m, r}(y)$ is a polynomial of degree $m$. Furthermore, we will represent (36) as linear combinations of Frobenius polynomials themselves (see Theorem 2) by using the well known inverse relations between signed Stirling numbers of the first kind and Stirling numbers of the second kind. We note here that Zhao and Chen [13] expressed $(y+1)^{r} \alpha_{m, r}(y)$ (see (77)) as linear combinations of Frobenius polynomials, which was obtained by repeatedly differentiating the generating function of Frobenius polynomials in (34). Moreover, they were able to determine the coefficients $C(r, i)$ appearing in (77) only recursively, not explicitly. These were determined explicitly by the above mentioned inverse relations between the two kinds of Stirling numbers.

We observe here that by taking $(r+1)$ th power of the equation in (34), the sum of products in (36) is explicitly given by

$$
\alpha_{m, r}(y)=\sum_{l=0}^{m}(r+l)_{l} S_{2}(m, l) y^{l}
$$

where $(x)_{n}$ are the falling factorial sequence defined in (1) (see [14,15]).

Before we close this section, we would like to recall some of the previous results related to the present work. In the same way as in this study, some sums of finite products of Chebyshev polynomials of the first, second, third and fourth kinds, and those of Legendre, Laguerre, Lucas and Fibonacci polynomials are expressed in terms of Chebyshev polynomials of all kinds (see [4,16-19]) and also in terms of Hermite, extended Laguerre, Legendre, Gegenbauer and Jacobi polynomials (see [20-23]).

In addition, those sums of finite products of all such polynomials can be expressed as linear combinations of Bernoulli polynomials. Indeed, all of these are obtained by deriving Fourier series expansions for the functions intimately related to such sums of finite products. For these, we let the reader refer to the papers $[24,25]$ and the references therein.

\section{Proof of Theorem 1}

The following theorem is our main result and obtained by explicit computations. 
Theorem 1. Let $m, r$ be nonnegative integers. Then we have the following identities:

$$
\begin{aligned}
\sum_{i_{1}+\cdots+i_{r+1}=} & \left(\begin{array}{c}
m \\
i_{1}, \cdots, i_{r+1}
\end{array}\right) F_{i_{1}}(y) \cdots F_{i_{r+1}}(y) \\
= & \frac{1}{r !} \sum_{k=0}^{m} \frac{\epsilon_{k}}{2^{k}} \sum_{s=0}^{\left[\frac{m-k}{2}\right]} \frac{(r+k+2 s) !(k+2 s) !}{4^{s}(s+k) ! s !} S_{2}(m, k+2 s) T_{k}(y) \\
= & \frac{1}{r !} \sum_{k=0}^{m} \frac{(k+1)}{2^{k}} \sum_{s=0}^{\left[\frac{m-k}{2}\right]} \frac{(r+k+2 s) !(k+2 s) !}{4^{s}(s+k+1) ! s !} S_{2}(m, k+2 s) U_{k}(y) \\
= & \frac{1}{r !} \sum_{k=0}^{m} \frac{1}{2^{k}} \sum_{s=0}^{m-k} \frac{(r+k+s) !(k+s) !}{2^{s}\left(k+\left[\frac{s}{2}\right]\right) !\left[\frac{s}{2}\right] !} S_{2}(m, k+s) V_{k}(y) \\
= & \frac{1}{r !} \sum_{k=0}^{m} \frac{1}{2^{k}} \sum_{s=0}^{m-k} \frac{(-1)^{s}(r+k+s) !(k+s) !}{2^{s}\left(k+\left[\frac{s}{2}\right]\right) !\left[\frac{s}{2}\right] !} S_{2}(m, k+s) W_{k}(y) \\
= & \frac{1}{r !} \sum_{k=0}^{m} \frac{1}{2^{k} k !} \sum_{s=0}^{\left[\frac{m-k}{2}\right]} \frac{(r+k+2 s) !(k+2 s) !}{4^{s} s !} S_{2}(m, k+2 s) H_{k}(y) \\
= & \frac{1}{r !} \sum_{k=0}^{m} \frac{(-1)^{k}}{\Gamma(\alpha+k+1)} \sum_{l=k}^{m} \frac{(r+l) ! l ! \Gamma(l+\alpha+1)}{(l-k) !} S_{2}(m, l) L_{k}^{\alpha}(y) \\
= & \frac{1}{r !} \sum_{k=0}^{m} 2^{k+1}(2 k+1) \sum_{s=0}^{\left[\frac{m-k}{2}\right]} \frac{(r+k+2 s) !(k+2 s) !(k+s+1) !}{s !(2 k+2 s+2) !} S_{2}(m, k+2 s) P_{k}(y) \\
= & \frac{\Gamma(\lambda)}{r !} \sum_{k=0}^{m} \frac{(k+\lambda)}{2^{k}} \sum_{s=0}^{\left[\frac{m-k}{2}\right]} \frac{(r+k+2 s) !(k+2 s) !}{4^{s} s ! \Gamma(s+k+\lambda+1)} S_{2}(m, k+2 s) C_{k}^{(\lambda)}(y) \\
= & \frac{1}{r !} \sum_{k=0}^{m} \frac{(-2)^{k} \Gamma(k+\alpha+\beta+1)}{\Gamma(2 k+\alpha+\beta+1)} \sum_{l=k}^{m} \frac{(-1)^{l}(r+l) ! l !}{(l-k) !} \\
& \times S_{2}(m, l){ }_{2} F_{1}(k-l, k+\beta+1 ; 2 k+\alpha+\beta+2 ; 2) P_{k}^{(\alpha, \beta)}(y) . \\
& (k+k)
\end{aligned}
$$

Here we are going to show Theorem 1 by using Propositions 1 and 2 which are stated in below.

Proposition 1. Let $q(x) \in \mathbb{R}[x]$ be a polynomial of degree $n$. Then the following hold true.

(a)

$$
\begin{gathered}
q(x)=\sum_{k=0}^{n} C_{k, 1} T_{k}(x), \text { where } \\
C_{k, 1}=\frac{(-1)^{k} 2^{k} k ! \epsilon_{k}}{(2 k) ! \pi} \int_{-1}^{1} q(x) \frac{d^{k}}{d x^{k}}\left(1-x^{2}\right)^{k-\frac{1}{2}} d x,
\end{gathered}
$$

(b)

$$
\begin{gathered}
q(x)=\sum_{k=0}^{n} C_{k, 2} U_{k}(x) \text {, where } \\
C_{k, 2}=\frac{(-1)^{k} 2^{k+1}(k+1) !}{(2 k+1) ! \pi} \int_{-1}^{1} q(x) \frac{d^{k}}{d x^{k}}\left(1-x^{2}\right)^{k+\frac{1}{2}} d x,
\end{gathered}
$$

(c)

$$
\begin{gathered}
q(x)=\sum_{k=0}^{n} C_{k, 3} V_{k}(x), \text { where } \\
C_{k, 3}=\frac{(-1)^{k} k ! 2^{k}}{(2 k) ! \pi} \int_{-1}^{1} q(x) \frac{d^{k}}{d x^{k}}(1-x)^{k-\frac{1}{2}}(1+x)^{k+\frac{1}{2}} d x,
\end{gathered}
$$


(d)

$$
\begin{gathered}
q(x)=\sum_{k=0}^{n} C_{k, 4} W_{k}(x), \text { where } \\
C_{k, 4}=\frac{(-1)^{k} k ! 2^{k}}{(2 k) ! \pi} \int_{-1}^{1} q(x) \frac{d^{k}}{d x^{k}}(1-x)^{k+\frac{1}{2}}(1+x)^{k-\frac{1}{2}} d x,
\end{gathered}
$$

(e)

$$
\begin{gathered}
q(x)=\sum_{k=0}^{n} C_{k, 5} H_{k}(x), \text { where } \\
C_{k, 5}=\frac{(-1)^{k}}{2^{k} k ! \sqrt{\pi}} \int_{-\infty}^{\infty} q(x) \frac{d^{k}}{d x^{k}} e^{-x^{2}} d x
\end{gathered}
$$

$(f)$

$$
\begin{gathered}
q(x)=\sum_{k=0}^{n} C_{k, 6} L_{k}^{\alpha}(x), \text { where } \\
C_{k, 6}=\frac{1}{\Gamma(\alpha+k+1)} \int_{0}^{\infty} q(x) \frac{d^{k}}{d x^{k}}\left(e^{-x} x^{k+\alpha}\right) d x,
\end{gathered}
$$

$(g)$

$$
\begin{gathered}
q(x)=\sum_{k=0}^{n} C_{k, 7} P_{k}(x), \text { where } \\
C_{k, 7}=\frac{2 k+1}{2^{k+1} k !} \int_{-1}^{1} q(x) \frac{d^{k}}{d x^{k}}\left(x^{2}-1\right)^{k} d x
\end{gathered}
$$

(h)

$$
\begin{gathered}
q(x)=\sum_{k=0}^{n} C_{k, 8} C_{k}^{(\lambda)}(x), \text { where } \\
C_{k, 8}=\frac{(k+\lambda) \Gamma(\lambda)}{(-2)^{k} \sqrt{\pi} \Gamma\left(k+\lambda+\frac{1}{2}\right)} \int_{-1}^{1} q(x) \frac{d^{k}}{d x^{k}}\left(1-x^{2}\right)^{k+\lambda-\frac{1}{2}} d x
\end{gathered}
$$

(i)

$$
\begin{gathered}
q(x)=\sum_{k=0}^{n} C_{k, 9} P_{k}^{(\alpha, \beta)}(x), \text { where } \\
C_{k, 9}=\frac{(-1)^{k}(2 k+\alpha+\beta+1) \Gamma(k+\alpha+\beta+1)}{2^{\alpha+\beta+k+1} \Gamma(\alpha+k+1) \Gamma(\beta+k+1)} \int_{-1}^{1} q(x) \frac{d^{k}}{d x^{k}}(1-x)^{k+\alpha}(1+x)^{k+\beta} d x .
\end{gathered}
$$

We note that the formulas (a), (b), (c), (d), (e), (f), (g), (h) and (i) of Proposition 1 are respectively from (24) of [4], (30) of [4], (23) of [3], (38) of [3], (3.7) of [6], (2.3) of [8], (2.3) of [7], (2.3) of [5] and (2.7) of [9]. In fact, all the facts can be derived from the orthogonalities in (24), (26)-(33), Rodrigues' and Rodrigues-type formulas in (15)-(23), and integration by parts.

Equations (a)-(d) and (e)-(h) of the next proposition are stated and proved respectively in $[23,26]$.

Proposition 2. Let $m$ and $k$ be any nonnegative integers. Then we have the following:

(a)

$$
\int_{-1}^{1}\left(1-x^{2}\right)^{k-\frac{1}{2}} x^{m} d x= \begin{cases}0, & \text { if } m \equiv 1(\bmod 2) \\ \frac{m !(2 k) ! \pi}{2^{m+2 k}\left(\frac{m}{2}+k\right) !\left(\frac{m}{2}\right) ! k !}, & \text { if } m \equiv 0(\bmod 2)\end{cases}
$$


(b)

$$
\int_{-1}^{1}\left(1-x^{2}\right)^{k+\frac{1}{2}} x^{m} d x= \begin{cases}0, & \text { if } m \equiv 1(\bmod 2) \\ \frac{m !(2 k+2) ! \pi}{2^{m+2 k+2}\left(\frac{m}{2}+k+1\right) !\left(\frac{m}{2}\right) !(k+1) !}, & \text { if } m \equiv 0(\bmod 2)\end{cases}
$$

(c)

$$
\int_{-1}^{1}(1-x)^{k-\frac{1}{2}}(1+x)^{k+\frac{1}{2}} x^{m} d x= \begin{cases}\frac{(m+1) !(2 k) ! \pi}{2^{m+2 k+1}\left(\frac{m+1}{2}+k\right) !\left(\frac{m+1}{2}\right) ! k !}, & \text { if } m \equiv 1(\bmod 2) \\ \frac{m !(2 k) ! \pi}{2^{m+2 k}\left(\frac{m}{2}+k\right) !\left(\frac{m}{2}\right) ! k !}, & \text { if } m \equiv 0(\bmod 2)\end{cases}
$$

(d)

$$
\int_{-1}^{1}(1-x)^{k+\frac{1}{2}}(1+x)^{k-\frac{1}{2}} x^{m} d x= \begin{cases}-\frac{(m+1) !(2 k) ! \pi}{2^{m+2 k+1}\left(\frac{m+1}{2}+k\right) !\left(\frac{m+1}{2}\right) ! k !^{\prime}}, & \text { if } m \equiv 1(\bmod 2) \\ \frac{m !(2 k) ! \pi}{2^{m+2 k}\left(\frac{m}{2}+k\right) !\left(\frac{m}{2}\right) ! k !}, & \text { if } m \equiv 0(\bmod 2)\end{cases}
$$

(e)

$$
\int_{-\infty}^{\infty} x^{m} e^{-x^{2}} d x= \begin{cases}0, & \text { if } m \equiv 1(\bmod 2) \\ \frac{m ! \sqrt{\pi}}{\left(\frac{m}{2}\right) ! 2^{m}}, & \text { if } m \equiv 0(\bmod 2)\end{cases}
$$

$(f)$

$$
\int_{-\infty}^{\infty} x^{m}\left(1-x^{2}\right)^{k} d x= \begin{cases}0, & \text { if } m \equiv 1(\bmod 2) \\ \frac{2^{2 k+2} k ! m !\left(k+\frac{m}{2}+1\right) !}{\left(\frac{m}{2}\right) !(2 k+m+2) !}, & \text { if } m \equiv 0(\bmod 2)\end{cases}
$$

$(g)$

$$
\int_{-1}^{1} x^{m}\left(1-x^{2}\right)^{k+\lambda-\frac{1}{2}} d x= \begin{cases}0, & \text { if } m \equiv 1(\bmod 2) \\ \frac{\Gamma\left(k+\lambda+\frac{1}{2}\right) \Gamma\left(\frac{m}{2}+\frac{1}{2}\right)}{\Gamma\left(k+\lambda+\frac{m}{2}+1\right)}, & \text { if } m \equiv 0(\bmod 2)\end{cases}
$$

(h)

$$
\begin{aligned}
\int_{-1}^{1} x^{m}(1-x)^{k+\alpha}(1+x)^{k+\beta} d x= & 2^{2 k+\alpha+\beta+1} \sum_{s=0}^{m}\left(\begin{array}{c}
m \\
s
\end{array}\right)(-1)^{m-s} 2^{s} \\
& \times \frac{\Gamma(k+\alpha+1) \Gamma(k+\beta+s+1)}{\Gamma(2 k+\alpha+\beta+s+2)} .
\end{aligned}
$$

Now, we will show (38), (40), (43), (45) and (46) in Theorem 1, while leaving the proofs of (39), (41), (42) and (44) to the reader.

With $\alpha_{m, r}(y)$ as in (36), let us put

$$
\alpha_{m, r}(y)=\sum_{k=0}^{m} C_{k, 1} T_{k}(y) .
$$


Then, by invoking (a) of Proposition 1, (a) of Proposition 2, (37), and by performing integration by parts $k$ times, we have

$$
\begin{aligned}
C_{k, 1} & =\frac{(-1)^{k} 2^{k} k ! \epsilon_{k}}{(2 k) ! \pi} \int_{-1}^{1} \alpha_{m, r}(y) \frac{d^{k}}{d y^{k}}\left(1-y^{2}\right)^{k-\frac{1}{2}} d y \\
& =\frac{(-1)^{k} 2^{k} k ! \epsilon_{k}}{(2 k) ! \pi} \sum_{l=0}^{m}(r+l)_{l} S_{2}(m, l) \int_{-1}^{1} y^{l} \frac{d^{k}}{d y^{k}}\left(1-y^{2}\right)^{k-\frac{1}{2}} d y \\
& =\frac{(-1)^{k} 2^{k} k ! \epsilon_{k}}{(2 k) ! \pi} \sum_{l=k}^{m}(r+l)_{l} S_{2}(m, l)(-1)^{k}(l)_{k} \int_{-1}^{1} y^{l-k}\left(1-y^{2}\right)^{k-\frac{1}{2}} d y,
\end{aligned}
$$

where

$$
\int_{-1}^{1} y^{l-k}\left(1-y^{2}\right)^{k-\frac{1}{2}} d y= \begin{cases}0, & \text { if } l \not \equiv k(\bmod 2) \\ \frac{(l-k) !(2 k) ! \pi}{2^{l+k}\left(\frac{l+k}{2}\right) !\left(\frac{l-k}{2}\right) ! k !}, & \text { if } l \equiv k(\bmod 2) .\end{cases}
$$

From (48), (49), replacing $l$ by $k+2 s$, and using $(x+r)_{r}=\frac{(x+r) !}{x !},\left(r \in \mathbb{Z}_{\geq 0}\right)$, we obtain

$$
\begin{aligned}
C_{k, 1} & =\frac{(-1)^{k} 2^{k} k ! \epsilon_{k}}{(2 k) ! \pi} \sum_{\substack{k \leq l \leq m \\
l \equiv k(m o d\\
}}(r+l)_{l} S_{2}(m, l)(-1)^{k}(l)_{k} \frac{(l-k) !(2 k) ! \pi}{2^{l+k}\left(\frac{l+k}{2}\right) !\left(\frac{l-k}{2}\right) ! k !} \\
& =\frac{(-1)^{k} 2^{k} k ! \epsilon_{k}}{(2 k) ! \pi} \sum_{s=0}^{\left[\frac{m-k}{2}\right]}(r+k+2 s)_{k+2 s} S_{2}(m, k+2 s)(-1)^{k}(k+2 s)_{k} \frac{(2 s) !(2 k) ! \pi}{2^{2 s+2 k}(s+k) ! s ! k !} \\
& =\frac{\epsilon_{k}}{r ! 2^{k}} \sum_{s=0}^{\left[\frac{m-k}{2}\right]} \frac{(r+k+2 s) !(k+2 s) !}{4^{s}(s+k) ! s !} S_{2}(m, k+2 s) .
\end{aligned}
$$

Thus, we have shown that

$$
\alpha_{m, r}(y)=\frac{1}{r !} \sum_{k=0}^{m} \frac{\epsilon_{k}}{2^{k}} \sum_{s=0}^{\left[\frac{m-k}{2}\right]} \frac{(r+k+2 s) !(k+2 s) !}{4^{s}(s+k) ! s !} S_{2}(m, k+2 s) T_{k}(y) .
$$

This verifies (38) in Theorem 1.

Next, we let

$$
\alpha_{m, r}(y)=\sum_{k=0}^{m} C_{k, 3} V_{k}(y) .
$$

Then, by making use of (c) in Proposition 1, (c) in Proposition 2, (37), and integration by parts $k$ times, we get

$$
C_{k, 3}=\frac{(-1)^{k} k ! 2^{k}}{(2 k) ! \pi} \sum_{l=k}^{m}(r+l)_{l} S_{2}(m, l)(-1)^{k}(l)_{k} \int_{-1}^{1} y^{l-k}(1-y)^{k-\frac{1}{2}}(1+y)^{k+\frac{1}{2}} d y,
$$

where

$$
\int_{-1}^{1} y^{l-k}(1-y)^{k-\frac{1}{2}}(1+y)^{k+\frac{1}{2}} d y= \begin{cases}\frac{(l-k+1) !(2 k) ! \pi}{2^{l+k+1}\left(\frac{l+k+1}{2}\right) !\left(\frac{l-k+1}{2}\right) ! k !}, & \text { if } l \not \equiv k(\bmod 2) \\ \frac{(l-k) !(2 k) ! \pi}{2^{l+k}\left(\frac{l+k}{2}\right) !\left(\frac{l-k}{2}\right) ! k !}, & \text { if } l \equiv k(\bmod 2) .\end{cases}
$$


To proceed further, we observe the following:

$$
\begin{aligned}
& \frac{(-1)^{k} k ! 2^{k}}{(2 k) ! \pi} \sum_{\substack{k \leq l \leq m \\
l \neq k(\bmod 2)}}(r+l)_{l} S_{2}(m, l)(-1)^{k}(l)_{k} \frac{(l-k+1) !(2 k) ! \pi}{2^{l+k+1}\left(\frac{l+k+1}{2}\right) !\left(\frac{l-k+1}{2}\right) ! k !} \\
&= \frac{(-1)^{k} k ! 2^{k}}{(2 k) ! \pi} \sum_{s=0}^{\left[\frac{m-k-1}{2}\right]}(r+k+2 s+1)_{k+2 s+1} S_{2}(m, k+2 s+1)(-1)^{k}(k+2 s+1)_{k} \\
& \times \frac{(2 s+2) !(2 k) ! \pi}{2^{2 s+2 k+2}(s+k+1) !(s+1) ! k !} \\
&= \frac{1}{r ! 2^{k}} \sum_{s=0}^{\left.\frac{m-k-1}{2}\right]} \frac{(r+k+2 s+1) !(k+2 s+1) !}{2^{2 s+1}(s+k+1) ! s !} S_{2}(m, k+2 s+1) \\
& \frac{(-1)^{k} k ! 2^{k}}{(2 k) ! \pi} \quad \sum_{\substack{k \leq l \leq m \\
l \equiv m(m o d}}(r+l){ }_{l} S_{2}(m, l)(-1)^{k}(l)_{k} \frac{(l-k) !(2 k) ! \pi}{2^{l+k}\left(\frac{l+k}{2}\right) !\left(\frac{l-k}{2}\right) ! k !} \\
&=\frac{(-1)^{k} k ! 2^{k}}{(2 k) ! \pi} \sum_{s=0}^{\left[\frac{m-k}{2}\right]}(r+k+2 s)_{k+2 s} S_{2}(m, k+2 s)(-1)^{k}(k+2 s)_{k} \frac{(2 s) !(2 k) ! \pi}{2^{2 k+2 s}(k+s) ! s ! k !} \\
&=\frac{1}{r ! 2^{k}} \sum_{s=0}^{\left[\frac{m-k}{2}\right]} \frac{(r+k+2 s) !(k+2 s) !}{2^{2 s}(k+s) ! s !} S_{2}(m, k+2 s) .
\end{aligned}
$$

Combining (51)-(55), we finally get

$$
\alpha_{m, r}(y)=\frac{1}{r !} \sum_{k=0}^{m} \frac{1}{2^{k}} \sum_{s=0}^{m-k} \frac{(r+k+s) !(k+s) !}{2^{s}\left(k+\left[\frac{s}{2}\right]\right) !\left[\frac{s}{2}\right] !} S_{2}(m, k+s) V_{k}(y) .
$$

This completes the proof for (40) in Theorem 1.

Next, let us put

$$
\alpha_{m, r}(y)=\sum_{k=0}^{m} C_{k, 6} L_{k}^{\alpha}(y) .
$$

Then, by (f) of Proposition 1, (37) and integration by parts $k$ times, we have

$$
\begin{aligned}
C_{k, 6} & =\frac{1}{\Gamma(\alpha+k+1)} \sum_{l=0}^{m}(r+l)_{l} S_{2}(m, l) \int_{0}^{\infty} y^{l} \frac{d^{k}}{d y^{k}}\left(e^{-y} y^{k+\alpha}\right) d y \\
& =\frac{1}{\Gamma(\alpha+k+1)} \sum_{l=k}^{m}(r+l)_{l} S_{2}(m, l)(-1)^{k}(l)_{k} \int_{0}^{\infty} e^{-y} y^{l+\alpha} d y \\
& =\frac{(-1)^{k}}{r ! \Gamma(\alpha+k+1)} \sum_{l=k}^{m} \frac{(r+l) ! l ! \Gamma(l+\alpha+1)}{(l-k) !} S_{2}(m, l) .
\end{aligned}
$$

Thus, we have shown that

$$
\alpha_{m, r}(y)=\frac{1}{r !} \sum_{k=0}^{m} \frac{(-1)^{k}}{\Gamma(\alpha+k+1)} \sum_{l=k}^{m} \frac{(r+l) ! l ! \Gamma(l+\alpha+1)}{(l-k) !} S_{2}(m, l) L_{k}^{\alpha}(y) .
$$

This verifies (43) in Theorem 1. Next, we let

$$
\alpha_{m, r}(y)=\sum_{k=0}^{m} C_{k, 8} C_{k}^{(\lambda)}(y)
$$


Then, from (h) of Proposition 1, (g) of Proposition 2, (37) and by integration by parts $k$ times, we obtain

$$
C_{k, 8}=\frac{(k+\lambda) \Gamma(\lambda)}{(-2)^{k} \sqrt{\pi} \Gamma\left(k+\lambda+\frac{1}{2}\right)} \sum_{l=k}^{m}(r+l)_{l} S_{2}(m, l)(-1)^{k}(l)_{k} \int_{-1}^{1} y^{l-k}\left(1-y^{2}\right)^{k+\lambda-\frac{1}{2}} d y,
$$

where

$$
\int_{-1}^{1} y^{l-k}\left(1-y^{2}\right)^{k+\lambda-\frac{1}{2}} d y= \begin{cases}0, & \text { if } l \not \equiv k(\bmod 2), \\ \frac{\Gamma\left(k+\lambda+\frac{1}{2}\right) \Gamma\left(\frac{l-k}{2}+\frac{1}{2}\right)}{\Gamma\left(\frac{+1 k}{2}+\lambda+1\right)}, & \text { if } l \equiv k(\bmod 2) .\end{cases}
$$

From (59), (60) and after some simplifications, we get

$$
\begin{aligned}
C_{k, 8} & =\frac{(k+\lambda) \Gamma(\lambda)}{2^{k} \sqrt{\pi}} \sum_{\substack{k \leq l \leq m \\
l \equiv k(\bmod 2)}}(r+l)_{l} S_{2}(m, l)(l)_{k} \frac{\Gamma\left(\frac{l-k+1}{2}\right)}{\Gamma\left(\frac{l+k}{2}+\lambda+1\right)} \\
& =\frac{(k+\lambda) \Gamma(\lambda)}{2^{k} \sqrt{\pi}} \sum_{s=0}^{\left[\frac{m-k}{2}\right]}(r+k+2 s)_{k+2 s} S_{2}(m, k+2 s)(k+2 s)_{k} \frac{\Gamma\left(s+\frac{1}{2}\right)}{\Gamma(k+s+\lambda+1)} \\
& =\frac{(k+\lambda) \Gamma(\lambda)}{r ! 2^{k}} \sum_{s=0}^{\left[\frac{m-k}{2}\right]} \frac{(r+k+2 s) !(k+2 s) !}{4^{s} s ! \Gamma(k+s+\lambda+1)} S_{2}(m, k+2 s) .
\end{aligned}
$$

Thus, we have shown that

$$
\alpha_{m, r}(y)=\frac{\Gamma(\lambda)}{r !} \sum_{k=0}^{m} \frac{(k+\lambda)}{2^{k}} \sum_{s=0}^{\left[\frac{m-k}{2}\right]} \frac{(r+k+2 s) !(k+2 s) !}{4^{s} s ! \Gamma(k+s+\lambda+1)} S_{2}(m, k+2 s) C_{k}^{(\lambda)}(y) .
$$

This proves (45) in Theorem 1.

Finally, let us put

$$
\alpha_{m, r}(y)=\sum_{k=0}^{m} C_{k, 9} P_{k}^{(\alpha, \beta)}(y) .
$$

Then, by (i) of Proposition 1, (h) of Proposition 2, (37) and integration by parts $k$ times, we have

$$
\begin{aligned}
C_{k, 9}= & \frac{(-1)^{k}(2 k+\alpha+\beta+1) \Gamma(k+\alpha+\beta+1)}{2^{\alpha+\beta+k+1} \Gamma(\alpha+k+1) \Gamma(\beta+k+1)} \\
& \quad \times \sum_{l=k}^{m}(r+l)_{l} S_{2}(m, l)(-1)^{k}(l)_{k} \int_{-1}^{1} y^{l-k}(1-y)^{k+\alpha}(1+y)^{k+\beta} d y,
\end{aligned}
$$

where

$$
\begin{aligned}
\int_{-1}^{1} y^{l-k}(1-y)^{k+\alpha}(1+y)^{k+\beta} d y= & 2^{2 k+\alpha+\beta+1} \sum_{s=0}^{l-k}\left(\begin{array}{c}
l-k \\
s
\end{array}\right)(-1)^{l-k-s} 2^{s} \\
& \times \frac{\Gamma(k+\alpha+1) \Gamma(k+\beta+s+1)}{\Gamma(2 k+\alpha+\beta+s+2)} .
\end{aligned}
$$


From (63) and (64), and after some simplifications,

$$
\begin{aligned}
C_{k, 9}= & \frac{(2 k+\alpha+\beta+1)(-2)^{k} \Gamma(k+\alpha+\beta+1)}{r ! \Gamma(k+\beta+1)} \\
& \times \sum_{l=k}^{m}(r+l) ! l !(-1)^{l} S_{2}(m, l) \sum_{s=0}^{l-k} \frac{(-2)^{s} \Gamma(k+\beta+s+1)}{s !(l-k-s) ! \Gamma(2 k+\alpha+\beta+s+2)} \\
= & \frac{(-2)^{k} \Gamma(k+\alpha+\beta+1)}{r ! \Gamma(2 k+\alpha+\beta+1)} \sum_{l=k}^{m} \frac{(r+l) ! l !(-1)^{l} S_{2}(m, l)}{(l-k) !} \sum_{s=0}^{l-k} \frac{2^{s}\langle k-l\rangle_{s}\langle k+\beta+1\rangle_{s}}{s !\langle 2 k+\alpha+\beta+2\rangle_{s}} \\
= & \frac{(-2)^{k} \Gamma(k+\alpha+\beta+1)}{r ! \Gamma(2 k+\alpha+\beta+1)} \sum_{l=k}^{m} \frac{(r+l) ! l !(-1)^{l} S_{2}(m, l)}{(l-k) !}{ }_{2} F_{1}(k-l, k+\beta+1 ; 2 k+\alpha+\beta+2 ; 2) .
\end{aligned}
$$

Therefore, we have shown that

$$
\begin{aligned}
\alpha_{m, r}(y)= & \frac{1}{r !} \sum_{k=0}^{m} \frac{(-2)^{k} \Gamma(k+\alpha+\beta+1)}{\Gamma(2 k+\alpha+\beta+1)} \\
& \times \sum_{l=k}^{m} \frac{(r+l) ! l !(-1)^{l} S_{2}(m, l)_{2} F_{1}(k-l, k+\beta+1 ; 2 k+\alpha+\beta+2 ; 2)}{(l-k) !} P_{k}^{(\alpha, \beta)}(y) .
\end{aligned}
$$

This completes the proof for (46) in Theorem 1.

Example 1. Here we will illustrate Theorem 1 in the case that $r=1, m=2$. We first recall the following values of the Stirling numbers of the second kind:

$$
S_{2}(0,0)=1, S_{2}(1,0)=0, S_{2}(1,1)=1, S_{2}(2,0)=0, S_{2}(2,1)=1, S_{2}(2,2)=1
$$

Then, from (37), we observe that

$$
F_{0}(y)=1, F_{1}(y)=y, F_{2}(y)=y+2 y^{2}
$$

Also, from (6), (10) and (12) we see that Chebyshev polynomials of the second kind, Hermite polynomials and Legendre polynomials with degrees $\leq 2$ are given by:

$$
\begin{aligned}
& \qquad \begin{array}{l}
T_{0}(y)=1, T_{1}(y)=y, T_{2}(y)=2 y^{2}-1, H_{0}(y)=1, H_{1}(y)=2 y, H_{2}(y)=4 y^{2}-2, \\
\qquad P_{0}(y)=1, P_{1}(y)=y, P_{2}(y)=\frac{1}{2}\left(3 y^{2}-1\right) .
\end{array} \\
& \text { For } r=1, m=2, \text { we have }
\end{aligned}
$$

$$
\begin{aligned}
\alpha_{2,1}(y) & =\sum_{i_{1}+i_{2}=2}\left(\begin{array}{c}
2 \\
i_{1}, i_{2}
\end{array}\right) F_{i_{1}}(y) F_{i_{2}}(y) \\
& =F_{2}(y) F_{0}(y)+2 F_{1}(y) F_{1}(y)+F_{0}(y) F_{2}(y) \\
& =2 y+6 y^{2} .
\end{aligned}
$$

From (38), (42) and (44), we have

$$
\begin{aligned}
\alpha_{2,1}(y) & =\sum_{k=0}^{2} \frac{\epsilon_{k}}{2^{k}} \sum_{s=0}^{\left[\frac{2-k}{2}\right]} \frac{(1+k+2 s) !(k+2 s) !}{4^{s}(s+k) ! s !} S_{2}(2, k+2 s) T_{k}(y) \\
& =S_{2}(2,0) T_{0}(y)+3 S_{2}(2,2) T_{0}(y)+2 S_{2}(2,1) T_{1}(y)+3 S_{2}(2,2) T_{2}(y) \\
& =2 y+6 y^{2}
\end{aligned}
$$




$$
\begin{aligned}
\alpha_{2,1}(y) & =\sum_{k=0}^{2} \frac{1}{2^{k} k !} \sum_{s=0}^{\left[\frac{2-k}{2}\right]} \frac{(1+k+2 s) !(k+2 s) !}{4^{s} s !} S_{2}(2, k+2 s) H_{k}(y) \\
& =S_{2}(2,0) H_{0}(y)+3 S_{2}(2,2) H_{0}(y)+S_{2}(2,1) H_{1}(y)+\frac{3}{2} S_{2}(2,2) H_{2}(y) \\
& =2 y+6 y^{2}, \\
\alpha_{2,1}(y)= & \sum_{k=0}^{2} 2^{k+1}(2 k+1) \sum_{s=0}^{\left[\frac{2-k}{2}\right]} \frac{(1+k+2 s) !(k+2 s) !(k+s+1) !}{s !(2 k+2 s+2) !} S_{2}(2, k+2 s) P_{k}(y) \\
= & S_{2}(2,0) P_{0}(y)+2 S_{2}(2,2) P_{0}(y)+2 S_{2}(2,1) P_{1}(y)+4 S_{2}(2,2) P_{2}(y) \\
= & 2 y+6 y^{2} .
\end{aligned}
$$

\section{Further Remarks}

Recall here that for any positive integer $r$, the Bernoulli numbers $B_{n}^{(r)}$ of order $r$ are given by

$$
\left(\frac{t}{e^{t}-1}\right)^{r}=\sum_{n=0}^{\infty} B_{n}^{(r)} \frac{t^{n}}{n !}
$$

The first identity (67) in the next proposition was noted earlier (see (36), (37)). Here we show the second identity (68).

Proposition 3. Let $n, r$ be any nonnegative integers. Then we have

$$
\begin{aligned}
\sum_{i_{1}+\cdots+B_{r+1}=n}\left(\begin{array}{c}
n \\
i_{1}, \cdots, i_{r+1}
\end{array}\right) F_{i_{1}}(y) \cdots F_{i_{r+1}}(y) & =\sum_{k=0}^{n}(r+k)_{k} S_{2}(n, k) y^{k} \\
& =\left(\frac{1}{r !}\right)^{2} \sum_{m=0}^{n} \frac{\left(\begin{array}{c}
n \\
m
\end{array}\right)}{\left(\begin{array}{c}
m+r \\
r
\end{array}\right)} B_{n-m}^{(r)} F_{m+r}^{(r)}(y) .
\end{aligned}
$$

Proof. By taking the $r^{\text {th }}$ derivative $\frac{\partial^{r}}{\partial y^{r}}$ on (34), we obtain

$$
r !\left(e^{t}-1\right)^{r}\left(1-y\left(e^{t}-1\right)\right)^{-(r+1)}=\sum_{m=0}^{\infty} F_{m+r}^{(r)}(y) \frac{t^{m+r}}{(m+r) !} .
$$

From (69), we have

$$
\begin{aligned}
\left(1-y\left(e^{t}-1\right)\right)^{-(r+1)} & =\frac{1}{r !}\left(\frac{t}{e^{t}-1}\right)^{r} \sum_{m=0}^{\infty} F_{m+r}^{(r)}(y) \frac{t^{m}}{(m+r) !} \\
& =\frac{1}{r !}\left(\sum_{l=0}^{\infty} B_{l}^{(r)} \frac{t^{l}}{l !}\right)\left(\sum_{m=0}^{\infty} \frac{m !}{(m+r) !} F_{m+r}^{(r)}(y) \frac{t^{m}}{m !}\right) \\
& =\frac{1}{r !} \sum_{n=0}^{\infty}\left(\sum_{m=0}^{n}\left(\begin{array}{c}
n \\
m
\end{array}\right) \frac{m !}{(m+r) !} B_{n-m}^{(r)} F_{m+r}^{(r)}(y)\right) \frac{t^{m}}{m !} \\
& =\left(\frac{1}{r !}\right)^{2} \sum_{n=0}^{\infty}\left(\sum_{m=0}^{n} \frac{\left(\begin{array}{c}
n \\
m
\end{array}\right)}{\left(\begin{array}{c}
m+r \\
r
\end{array}\right)} B_{n-m}^{(r)} F_{m+r}^{(r)}(y)\right) \frac{t^{n}}{n !} .
\end{aligned}
$$

Corollary 1. Let $n, l, r$ be any integers with $0 \leq l \leq n, r \geq 1$. Then we have the following identity.

$$
S_{2}(n, l)=\left(\begin{array}{c}
l+r \\
r
\end{array}\right) \sum_{m=l}^{n} \frac{\left(\begin{array}{c}
n \\
m
\end{array}\right)}{\left(\begin{array}{c}
m+r \\
r
\end{array}\right)} B_{n-m}^{(r)} S_{2}(m+r, l+r) .
$$


Proof. First, from (35) we note that

$$
F_{m+r}^{(r)}(y)=\sum_{l=0}^{m}(l+r) ! S_{2}(m+r, l+r)(l+r)_{r} y^{l} .
$$

Substituting (71) into (68), and changing the order of summation, we get

$$
\sum_{l=0}^{n} \sum_{m=l}^{n} \frac{((l+r) !)^{2}}{(r !)^{2} l !} \frac{\left(\begin{array}{c}
n \\
m
\end{array}\right)}{\left(\begin{array}{c}
m+r \\
r
\end{array}\right)} B_{n-m}^{(r)} S_{2}(m+r, l+r) y^{l} .
$$

Comparison of the right hand side of (67) and (72) yields the result.

Remark 1. Taking $r=1$ in (70) gives us the following identity:

$$
S_{2}(n, l)=\frac{l+1}{n+1} \sum_{m=l}^{n}\left(\begin{array}{c}
n+1 \\
m+1
\end{array}\right) B_{n-m} S_{2}(m+1, l+1), \quad(0 \leq l \leq n) .
$$

As a check, we illustrate this for $n=7, l=3$. First, we recall the following values of Stirling numbers of the second kind and Bernoulli numbers

$$
\begin{aligned}
& S_{2}(7,3)=301, S_{2}(4,4)=1, S_{2}(5,4)=10, S_{2}(6,4)=65, S_{2}(7,4)=350, S_{2}(8,4)=1701, \\
& B_{0}=1, B_{1}=-\frac{1}{2}, B_{2}=\frac{1}{6}, B_{3}=0, B_{4}=-\frac{1}{30} .
\end{aligned}
$$

Then, we check that

$$
\begin{aligned}
\frac{3+1}{7+1} \sum_{m=3}^{7}\left(\begin{array}{l}
7+1 \\
m+1
\end{array}\right) B_{7-m} S_{2}(m+1,4) \\
\quad=\frac{1}{2}\left\{\left(\begin{array}{l}
8 \\
4
\end{array}\right) B_{4} S_{2}(4,4)+\left(\begin{array}{l}
8 \\
5
\end{array}\right) B_{3} S_{2}(5,4)+\left(\begin{array}{l}
8 \\
6
\end{array}\right) B_{2} S_{2}(6,4)+\left(\begin{array}{l}
8 \\
7
\end{array}\right) B_{1} S_{2}(7,4)+\left(\begin{array}{l}
8 \\
8
\end{array}\right) B_{0} S_{2}(8,4)\right\} \\
\quad=\frac{1}{2}\left\{70 \cdot\left(-\frac{1}{30}\right) \cdot 1+56 \cdot 0 \cdot 10+28 \cdot \frac{1}{6} \cdot 65+8 \cdot\left(-\frac{1}{2}\right) \cdot 350+1 \cdot 1 \cdot 1701\right\}=301
\end{aligned}
$$

As is well known, for any sequences $\left\{a_{m}\right\}_{m=0}^{\infty},\left\{b_{m}\right\}_{m=0}^{\infty}$ of complex numbers, we have:

$$
a_{k}=\sum_{l=0}^{n} S_{2}(l, k) b_{l},(0 \leq k \leq n) \text { if and only if } b_{k}=\sum_{l=0}^{n} S_{1}(l, k) a_{l},(0 \leq k \leq n),
$$

where $S_{1}(l, k)$ are the signed Stirling numbers of the first kind.

Next, we would like to express the sums of finite products of Fubini polynomials in the left hand side of (67) as linear combinations of Fubini polynomials.

Theorem 2. Let $n, r$ be any nonnegative integers. Then we have

$$
\sum_{i_{1}+\cdots+i_{r+1}=n}\left(\begin{array}{c}
n \\
i_{1}, \cdots, i_{r+1}
\end{array}\right) F_{i_{1}}(y) \cdots F_{i_{r+1}}(y)=\sum_{k=0}^{n} \sum_{l=k}^{n}\left(\begin{array}{c}
r+l \\
r
\end{array}\right) S_{1}(l, k) S_{2}(n, l) F_{k}(y) .
$$

Proof. Let us put

$$
\sum_{i_{1}+\cdots+i_{r+1}=n}\left(\begin{array}{c}
n \\
i_{1}, \cdots, i_{r+1}
\end{array}\right) F_{i_{1}}(y) \cdots F_{i_{r+1}}(y)=\sum_{l=0}^{n} C_{l} F_{l}(y) .
$$


Then, from the first identity in Proposition 3 and (35), we get

$$
\sum_{k=0}^{n}(r+k)_{k} S_{2}(n, k) y^{k}=\sum_{k=0}^{n}\left(\sum_{l=k}^{n} C_{l} S_{2}(l, k) k !\right) y^{k}
$$

By comparing the coefficients of (75), we obtain

$$
\left(\begin{array}{c}
r+k \\
r
\end{array}\right) S_{2}(n, k)=\sum_{l=0}^{n} C_{l} S_{2}(l, k), \quad(0 \leq k \leq n) .
$$

Now, the application of (73) to (76) gives us

$$
\begin{aligned}
C_{k} & =\sum_{l=0}^{n}\left(\begin{array}{c}
r+l \\
r
\end{array}\right) S_{1}(l, k) S_{2}(n, l) \\
& =\sum_{l=k}^{n}\left(\begin{array}{c}
r+l \\
r
\end{array}\right) S_{1}(l, k) S_{2}(n, l), \quad(0 \leq k \leq n),
\end{aligned}
$$

which completes the proof.

In [13], it was shown that for any nonnegative integers $n, r$, the following identity holds:

$$
\sum_{i_{1}+\cdots+i_{r+1}=n}\left(\begin{array}{c}
n \\
i_{1}, \cdots, i_{r+1}
\end{array}\right) F_{i_{1}}(y) \cdots F_{i_{r+1}}(y)=\frac{1}{r !(y+1)^{r}} \sum_{i=0}^{r} C(r, i) F_{n+r-i}(y),
$$

where $C(r, i),(0 \leq i \leq r)$ are determined by $C(r, 0)=1, C(r, r)=r !$, and

$$
C(r+1, k+1)=C(r, k+1)+(r+1) C(r, k), \quad(0 \leq k \leq r) .
$$

Here and below, we understand that $C(r, k)=0$, if $k>r$.

We note here, in passing, that an identity similar to (77) was derived for two variable Fubini polynomials in [10] (see also [14,15,27]).

Taking $n=0$ in (77) gives us

$$
r !(y+1)^{r}=\sum_{i=0}^{r} C(r, i) F_{r-i}(y) .
$$

By making use of (35), we obtain

$$
r ! \sum_{k=0}^{r}\left(\begin{array}{l}
r \\
k
\end{array}\right) y^{k}=\sum_{k=0}^{r}\left(\sum_{i=0}^{r-k} C(r, i) k ! S_{2}(r-i, k)\right) y^{k} .
$$

Comparing both sides of (79), we have

$$
\begin{aligned}
\frac{r !}{k !}\left(\begin{array}{l}
r \\
k
\end{array}\right) & =\sum_{i=0}^{r-k} S_{2}(r-i, k) C(r, i) \\
& =\sum_{i=0}^{r} S_{2}(r-i, k) C(r, i) \\
& =\sum_{i=0}^{r} S_{2}(i, k) C(r, r-i) .
\end{aligned}
$$


Again, an application of (73) to (80) yields

$$
C(r, r-k)=\sum_{i=0}^{r} \frac{r !}{i !}\left(\begin{array}{l}
r \\
i
\end{array}\right) S_{1}(i, k),
$$

or equivalently,

$$
\begin{aligned}
C(r, k) & =\sum_{i=0}^{r} \frac{r !}{i !}\left(\begin{array}{l}
r \\
i
\end{array}\right) S_{1}(i, r-k) \\
& =\sum_{i=r-k}^{r} \frac{r !}{i !}\left(\begin{array}{l}
r \\
i
\end{array}\right) S_{1}(i, r-k) .
\end{aligned}
$$

Substituting (81) into (77), we obtain the following expression:

$$
\begin{aligned}
& \sum_{i_{1}+\cdots+i_{r+1}=n}\left(\begin{array}{c}
n \\
i_{1}, \cdots, i_{r+1}
\end{array}\right) F_{i_{1}}(y) \cdots F_{i_{r+1}}(y) \\
& =\frac{1}{r !(y+1)^{r}} \sum_{i=0}^{r} \sum_{k=r-i}^{r} \frac{r !}{k !}\left(\begin{array}{l}
r \\
k
\end{array}\right) S_{1}(k, r-i) F_{n+r-i}(y) .
\end{aligned}
$$

\section{Conclusions}

In this paper, we considered the sums of finite products of Fubini polynomials $\alpha_{m, r}(y)$ in (36), and expressed each of them as linear combinations of $T_{n}(y), U_{n}(y), V_{n}(y), W_{n}(y), H_{n}(y), L_{n}^{\alpha}(y), P_{n}(y)$, $C_{n}^{(\lambda)}(y)$, and $P_{n}^{(\alpha, \beta)}(y)$ by performing explicit computations. Here we used the expression of $\alpha_{m, r}(y)$ in (37). We could have exploited the expression of $\alpha_{m, r}(y)$ in (68). However, it would have yielded more complicated expressions than the ones in Theorem 1. Then our results were obtained, in addition to (37), by using Propositions 1 and 2, and integration by parts. As a corollary to Proposition 3, we were able to discover the identity involving Stirling numbers of the second kind and higher order Bernoulli numbers which gives an interesting identity already in the special case of ordinary Bernoulli numbers (see Corollary 1, Remark 1). We also expressed $\alpha_{m, r}(y)$ as linear combinations of Fubini polynomials by using the well known inverse relations between signed Stirling numbers of the first kind and Stirling numbers of the second kind in (73) (see Theorem 2). Finally, we remarked that by applying the same inverse relations, we can give explicit expressions for the coefficients $C(r, i)$ appearing in the identity (77) that has been recently obtained by Zhao and Chen (see [13]) and determined only recursively. Along the same line as the present paper, we will continue to study sums of finite products of some interesting special polynomials and numbers and to find their applications to mathematics, physics and engineering, etc.

Author Contributions: Conceptualization, T.K.; Formal analysis, D.S.K. and T.K.; Funding acquisition, D.K.; Investigation, D.S.K., D.V.D., D.K. and T.K.; Methodology, T.K.; Supervision, D.S.K. and T.K.; Visualization, D.S.K., D.V.D., D.K. and T.K.; Writing—original draft, T.K.; Writing—review \& editing, D.S.K., D.V.D., D.K. and T.K.

Funding: This research received no external funding.

Conflicts of Interest: The authors declare no conflict of interest.

\section{References}

1. Andrews, G.E.; Askey, R.; Roy, R. Special functions. In Encyclopedia of Mathematics and Its Applications; Series Number 71; Cambridge University Press: Cambrige, UK, 1999.

2. Beals, R.; Wong, R. Special functions and orthogonal polynomials. In Cambridge Studies in Advanced Mathematics; Series Number 153; Cambridge University Press: Cambrige, UK, 2016.

3. Kim, D.S.; Dolgy, D.V.; Kim, T.; Rim, S.-H. Identities involving Bernoulli and Euler polynomials arising from Chebyshev polynomials. Proc. Jangjeon Math. Soc. 2012, 15, 361-370.

4. Kim, D.S.; Kim, T.; Lee, S.-H. Some identities for Bernoulli polynomials involving Chebyshev polynomials. J. Comput. Anal. Appl. 2014, 16, 172-180. 
5. Kim, D.S.; Kim, T.; Rim, S.-H. Some identities involving Gegenbauer polynomials. Adv. Differ. Equ. 2012, 2012, 219. [CrossRef]

6. Kim, D.S.; Kim, T.; Rim, S.-H.; Lee, S.-H. Hermite polynomials and their applications associated with Bernoulli and Euler numbers. Discret. Dyn. Nat. Soc. 2012, 2012, 974632. [CrossRef]

7. Kim, D.S.; Rim, S.-H.; Kim, T. Some identities on Bernoulli and Euler polynomials arising from orthogonality of Legendre polynomials. J. Inequal. Appl. 2012, 2012, 227. [CrossRef]

8. Kim, T.; Kim, D.S. Extended Laguerre polynomials associated with Hermite, Bernoulli, and Euler numbers and polynomials. Abstr. Appl. Anal. 2012, 2012, 957350. [CrossRef]

9. Kim, T.; Kim, D.S.; Dolgy, D.V. Some identities on Bernoulli and Hermite polynomials associated with Jacobi polynomials. Discret. Dyn. Nat. Soc. 2012, 2012, 584643.

10. Chen, G.; Chen, L. Some identities involving the Fubini polynomials and Euler polynomials. Mathematics 2018, 6, 300. [CrossRef]

11. Kargin, L. Some formulae for products of Fubini polynomials with applications. arXiv 2016, arXiv:1701.01023v1.

12. Kim, T.; Kim, D.S.; Jang, G.-W.; Kwon, J. Symmetric identities for Fubini polynomials. Symmetry 2018, 10, 219. [CrossRef]

13. Zhao, J.; Chen, Z. Some symmetric identities involving Fubini polynomials and Euler numbers. Symmetry 2018, 10, 303.

14. Jang, G.-W.; Dolgy, D.V.; Jang, L.-C.; Kim, D.S.; Kim, T. Sums of products of two variable higher-order Fubini functions arising from Fourier series. Adv. Stud. Contemp. Math. 2018, 28, 533-550.

15. Kim, D.S.; Kim, T.; Kwon, H.-I.; Park, J.-W. Two variable higher-order Fubini polynomials. J. Korean Math. Soc. 2018, 55, 975-986.

16. Kim, T.; Kim, D.S.; Dolgy, D.V.; Kwon, J. Representing sums of finite products of Chebyshev polynomials of the first kind and Lucas polynomials by Chebyshev polynomials. Mathematics 2019, 7, 26. [CrossRef]

17. Kim, T.; Kim, D.S.; Dolgy, V.; Ryoo, S. Representing sums of finite products of Chebyshev polynomials of third and fourth kinds by Chebyshev polynomials. Symmetry 2018, 10, 258. [CrossRef]

18. Kim, T.; Kim, D.S.; Jang, L.-C.; Dolgy, D.V. Representation by Chebyshev polynomials for sums of finite products of Chebyshev polynomials. Symmetry 2018, 10, 742. [CrossRef]

19. Kim, T.; Kim, D.S.; Kwon, J.; Jang, G.-W. Sums of finite products of Legendre and Laguerre polynomials by Chebyshev polynomials. Adv. Stud. Contemp. Math. 2018, 28, 551-565.

20. Dolgy, D.V.; Kim, D.S.; Kim, T.; Kwon, J. Connection problem for sums of finite products of Chebyshev polynomials of the third and fourth kinds. Symmetry 2018, 10, 617. [CrossRef]

21. Kim, T.; Hwang, K.-W.; Kim, D.S.; Dolgy, D.V. Connection problem for sums of finite products of Legendre and Laguerre polynomials. Symmetry 2018, 11, 317. [CrossRef]

22. Kim, T.; Kim, D.S.; Dolgy, D.V.; Kim, D. Representation by several orthogonal polynomials for sums of finite products of Chebyshev polynomials of the first, third and fourth kinds. Adv. Differ. Equ. 2019, $2019,110$. [CrossRef]

23. Kim, T.; Kim, D.S.; Kwon, J.; Dolgy, D.V. Expressing sums of finite products of Chebyshev polynomials of the second kind and of Fibonacci polynomials by several orthogonal polynomials. Mathematics 2018, 6, 210. [CrossRef]

24. Kim, T.; Kim, D.S.; Dolgy, V.; Park, J.-W. Sums of finite products of Chebyshev polynomials of the second kind and of Fibonacci polynomials. J. Inequal. Appl. 2018, 2018, 148. [CrossRef] [PubMed]

25. Kim, T.; Kim, D.S.; Jang, L.-C.; Jang, G.-W. Fourier series for functions related to Chebyshev polynomials of the first kind and Lucas polynomials. Mathematics 2018, 6, 276. [CrossRef]

26. Kim, T.; Dolgy, D.V.; Kim, D.S. Representing sums of finite products of Chebyshev polynomials of the second kind and Fibonacci polynomials in terms of Chebyshev polynomials. Adv. Stud. Contemp. Math. 2018, 28, 321-335.

27. Kim, T.; Kim, D.S.; Dolgy, D.V.; Jang, G.-W.; Kwon, J. Fourier series of functions related to two variable higher-order Fubini polynomials. Adv. Stud. Contemp. Math. 2018, 28, 589-605.

(c) 2019 by the authors. Licensee MDPI, Basel, Switzerland. This article is an open access article distributed under the terms and conditions of the Creative Commons Attribution (CC BY) license (http://creativecommons.org/licenses/by/4.0/). 